\title{
Polyelectrolyte Condensation Induced by Linear Cations
}

\author{
Camilo Guáqueta and Erik Luijten* \\ Department of Materials Science and Engineering, \\ University of Illinois at Urbana-Champaign, Urbana, Illinois 61801
}

(Dated: November 17, 2018)

\begin{abstract}
We examine the role of the condensing agent in the formation of polyelectrolyte bundles, via grandcanonical Monte Carlo simulations. Following recent experiments we use linear, rigid divalent cations of various lengths to induce condensation. Our results clarify and explain the experimental results for short cations. For longer cations we observe novel condensation behavior owing to alignment of the cations. We also study the role of the polyelectrolyte surface charge density, and find a nonmonotonic variation in bundle stability. This nonmonotonicity captures two trends that have been observed in separate experiments.

PACS numbers: 82.35.Rs, 87.15.Aa, 87.16.Ka
\end{abstract}

Under conditions commonly found in biological systems, like-charged polyelectrolytes in solution can form compact aggregates. This phenomenon, referred to as polyelectrolyte condensation, occurs for a variety of biopolymers, generally under the influence of charged condensing agents such as multivalent ions [1, 2], short polyamines [3], or charged proteins [4]. If the polyelectrolytes are stiff chains, such as filamentous actin (Factin) 1, 4] or the fd and M13 virus [2], they aggregate into dense, hexagonally coordinated bundles. The counterintuitive nature of the effective attraction implied by bundle formation, in combination with the biological importance of the phenomenon, have made it the topic of numerous studies over the past decades (see Refs. [5, 6, 7, 8, 9, 10] and references therein). There is now general agreement that strong electrostatic correlations are a crucial condition [9, 11], in accordance with the experimental observation that most condensing agents carry a multivalent charge. However, other factors that affect the tendency of a system to exhibit condensation are much less well established, mainly because these factors are difficult to disentangle in both experiments and theory.

Even for a simple ionic condensing agent, its interaction strength with the polyelectrolyte is affected not only by its valency, but also by its size [12] and by the surface charge density of the polyelectrolyte. In addition, the ionic concentration plays an important role, as it determines the stabilizing osmotic pressure exerted on the bundle by the surrounding solution [2, 4] and also controls the strength of entropic effects such as counterion release and depletion interactions [5]. Thus, even a systematic variation of the ion size can yield results that are difficult to interpret, as it alters both the binding of the ion to the polyelectrolyte and the osmotic pressure of the solution. Likewise, variation of the polyelectrolyte charge not only affects ionic binding, but also the direct electrostatic repulsion between polyelectrolytes. Accordingly, only an integrated approach can resolve the true origin of observed trends in the aggregation behavior.
There is a remarkable dearth of such studies. Experimentally, there are technical limitations. For example, examination of the effect of counterion size is hampered by the uncertainty in measuring hydrated ion sizes 13 and variation of counterion valency often entails the simultaneous variation of other ionic properties. Furthermore, it is difficult to measure the ionic concentration within the aggregate and hence to assess the osmotic effects arising from a concentration imbalance with the bulk solution. This osmotic stress is also often ignored in computational and theoretical studies. Inspired by two recent experimental studies [13, 14], in this Letter we aim to obtain a more complete understanding of how bundle formation is affected by (i) the size of the condensing agent and (ii) the surface charge density of the polyelectrolyte. In Ref. [14], the M13 virus was bundled using diamine molecules. In solution the diamines form divalent cations with a length that can be systematically varied. It was found that only the shortest diamines can induce bundle formation. This implies that increasing the diamine size decreases bundle stability, although a detailed analysis of the role of diamine size could not be obtained. Furthermore, it was found that increasing the M13 surface charge density $\sigma$ also destabilizes the bundle. In contrast, in Ref. [13] this virus was bundled using alkali earth metal ions and an increased stability was observed upon increase of the surface charge density [15]. It is our purpose to clarify these experimental findings through computer simulations. Indeed, earlier simulations [13] confirmed part of the observations, but did not explain the surface-charge dependence observed in Ref. 14] and did not address effects arising from internal degrees of freedom of the condensing agent. We confirm that an increase of the diamine size destabilizes the bundle, but also demonstrate how stability is recovered for even longer diamines through changes in their spatial arrangement. Furthermore, we reconcile the contradictory experimental findings for the effect of surface charge density. As the underlying mechanisms are highly generic, our findings are relevant for broad classes of sys- 


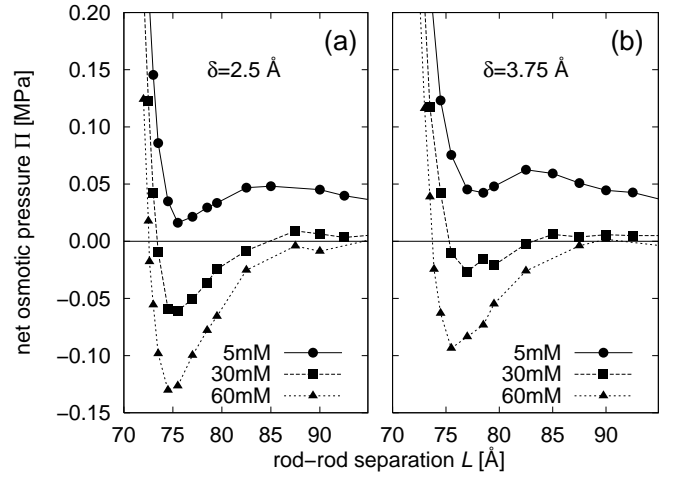

FIG. 1: Net osmotic pressure as a function of rod-rod separation $L$, for bulk diamine concentrations of 5,30 , and $60 \mathrm{mM}$, at a polyelectrolyte surface charge density $\sigma=0.633 \mathrm{e} / \mathrm{nm}^{2}$. The diamine charge separation is (a) $\delta=2.5 \AA$, and (b) $\delta=$ $3.75 \AA$. Error bars are comparable to the symbol size.

tems that display electrostatically induced aggregation.

We employ Monte Carlo simulations of a model based upon the experimental systems [13, 14]. M13 is modeled as an infinitely long cylindrical rod, with monovalent charges placed on a rectangular grid wrapped around the rod at a radial distance of $28 \AA$. The surface charge density is controlled via the lattice parameters of the grid. Soft repulsive $1 / r^{12}$ interactions between the cylinder and other particles bring the effective cylinder radius to $R=30.5 \AA$. The diamines are modeled as rigid straight molecules composed of soft repulsive beads of effective diameter $d=5 \AA$. The terminal beads, both carrying a monovalent charge, have a center-to-center separation $\delta$ and are connected by $\lceil\delta / d\rceil-1$ regularly spaced uncharged beads. The coions are monovalent beads of the same diameter. Water is represented as a homogeneous dielectric medium $(\varepsilon=80)$ and the temperature is set to $T=298 \mathrm{~K}$. Electrostatic interactions are calculated via Ewald summation. The polyelectrolytes are placed in a periodic cell, forming an infinite hexagonal array with center-to-center rod-rod separation $L$. To reduce finite-size effects a $2 \times 2$ array of rods is used. Rod degrees of freedom are ignored. As in Refs. 2, 13], we employ the grand-canonical ensemble to ensure that the bundle is in chemical equilibrium with a bulk solution of diamine salt (diamines plus coions). Thus, in addition to a fixed number (300-500) of diamines that balance the polyelectrolyte charge, the bundle contains a fluctuating amount of diamine salt. We perform separate simulations of the bulk solution to establish its osmotic pressure $\Pi_{\text {bulk }}$ as a function of chemical potential. Mechanical equilibrium is then obtained if the net osmotic pressure $\Pi=\left(\Pi_{\text {bundle }}-\Pi_{\text {bulk }}\right)$ vanishes.

Figure 1(a) shows the net osmotic pressure as a function of rod-rod separation, for diamines with length $\delta=2.5 \AA$, at three bulk diamine concentrations. Negative $\Pi$ corresponds to bundle contraction; a zero-crossing

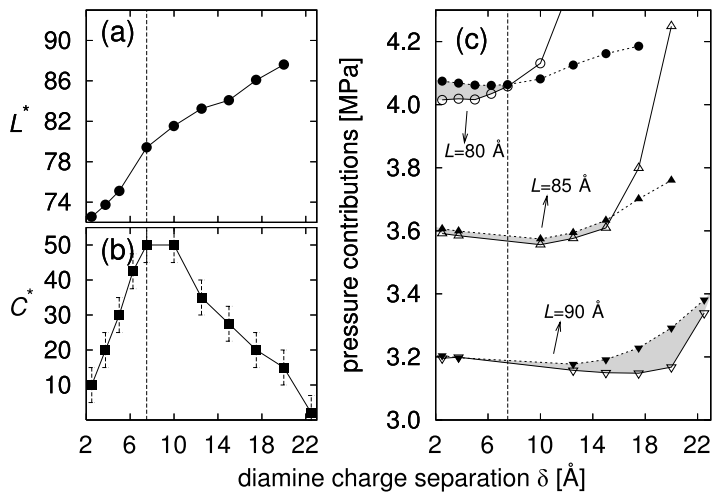

FIG. 2: Effect of diamine charge separation on bundle stability for polyelectrolytes with $\sigma=0.633 \mathrm{e} / \mathrm{nm}^{2}$. (a) Stable rod-rod separation at fixed $60 \mathrm{mM}$ diamine concentration. (b) Bulk diamine concentration $C^{*}$ at which bundling first occurs. (c) Separate contributions to the net osmotic pressure, for $L=80,85$, and $90 \AA$, at fixed $60 \mathrm{mM}$ diamine concentration. The dashed curves give the negative of the electrostatic contribution (i.e., electrostatic attraction), and the solid curves give the short-range contribution to the pressure.

at small $L$ indicates a free-energy minimum and yields the rod separation of a stable bundle. At low diamine concentrations $\Pi$ is always positive and no condensation takes place, but as the concentration exceeds a threshold value the pressure crosses zero and bundles form. This agrees with experimental results on M13 and fd [13, 14], and highlights the importance of the excess bulk diamine concentration. Experimentally [14], it is found that the bundles become unstable when $\delta$ increases. This is also reproduced by our simulations: for $\delta=3.75 \AA$ [Fig. 1(b)] the osmotic pressure curves are shifted outwards and upwards, reflecting a decrease in stability. To study this in detail, we vary the diamine length over a much wider range, $2.5 \AA \leq \delta \leq 22.5 \AA$. Surprisingly, two distinct regimes emerge. The trend observed experimentally and pictured in Fig. 1 continues up to $\delta \approx 7.5 \AA$, but for larger diamine lengths - not studied in Ref. 14] - this trend reverses and bundle stability increases with $\delta$.

To quantify and understand this behavior we refer to Fig. 2. The stable rod separation $L^{*}$ (at fixed, sufficiently high diamine concentration) is shown vs. diamine length in Fig. 2(a), and the threshold diamine concentration $C^{*}$ required for condensation [16] in Fig. 2(b). In both panels, a vertical line separates the two regimes. For smaller diamines, $\delta \lesssim 7.5 \AA$, the bundle swells linearly with $\delta$. This is accompanied by an increase in $C^{*}$, i.e., a larger bulk osmotic pressure is needed to maintain bundle stability. To understand this decrease in stability it is instructive to consider how the partitioning of diamines between the bundle and the bulk changes when $\delta$ increases [4]. On the one hand, larger diamines are more likely to be excluded from the bundle, thus enhancing the stabilizing effect of the bulk solution. On the other 


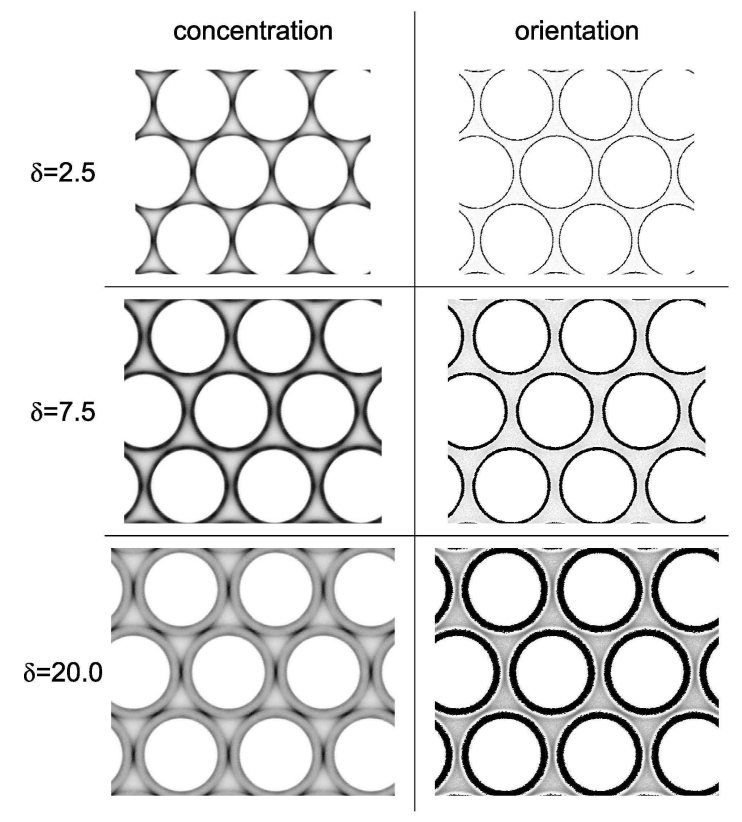

FIG. 3: Fluid structure within stable bundles, projected onto a plane perpendicular to the bundle axis, for three different diamine lengths (top to bottom: $\delta=2.5,7.5$, and $20 \AA$ ). Left: diamine concentration; right: diamine orientation with respect to polyelectrolyte axis. For discussion see the text.

hand, swelling allows more diamine salt to enter the bundle, decreasing the concentration difference. We find that the latter effect dominates: as $\delta$ increases from $2.5 \AA$ to $7.5 \AA$ the diamine salt concentration of in the bundle almost doubles. Accordingly, the bulk solution becomes less effective at holding the bundle together.

What drives the swelling of the bundle? Wong et al. [14] propose that longer diamines behave effectively as two monovalent ions rather than a single divalent unit, with the consequent loss of electrostatic attraction leading to dissolution of the bundle. We examine this idea in Fig. 2(c), which shows how the separate contributions to the net osmotic pressure vary with diamine size. Three different rod separations are plotted, spanning the range of Fig. 2(a). The solid curves (open symbols) show the short-range contribution to the pressure, which includes the kinetic and excluded-volume terms, and the dashed curves (filled symbols) show the negative of the electrostatic contribution. Thus, the intersection of two curves corresponds to $\Pi=0$, i.e., a stable bundle for the given $\delta$, and in the shaded regions $\Pi$ is negative. The top pair of curves $(L=80 \AA)$ is typical for the regime $\delta \lesssim 7.5 \AA$. Rather than the suggested loss of electrostatic attraction [14] we see that the electrostatic contribution is relatively constant, and it is instead the short-range repulsion that changes, rising sharply with $\delta$ and causing the bundle to swell and lose stability.

We now turn to the regime of large $\delta$. Near $\delta=7.5 \AA$ there is a weak change in slope in Fig. 2(a), indicat- ing a slower swelling of the bundle. More importantly, the threshold diamine concentration starts to decrease [Fig. 2(b)]. This reversal of $C^{*}$ is particularly remarkable in view of the continued swelling of the bundle, as it implies that bundle stability increases despite the weakening of the salt imbalance. Indeed, Fig. 22(c) shows that at large $\delta$ the electrostatic attraction no longer remains constant, but instead rises. Important insights into this phenomenon can be obtained from the distribution and orientation of diamines in the bundle, as shown in Fig. 3 for fixed bulk concentration. The three rows correspond to $\delta=2.5,7.5$, and $20 \AA$, each shown at the corresponding stable rod-rod separation. The left-hand column shows the spatial distribution of diamines in a cross-section of the bundle, with darker shading corresponding to higher concentration. The right-hand column shows how the diamines are oriented with respect to the rods, with black indicating a tendency to align parallel to the rods (outof-plane), grey indicating perpendicular alignment (inplane), and white indicating isotropic orientation. For small diamines $(\delta=2.5 \AA)$ the bundle is quite compact, and the diamines are concentrated mostly in the two-fold bridging sites between pairs of rods. The orientation of the diamines is essentially isotropic. At $\delta=7.5 \AA-$ where the bundle is swollen and has a low stability - the diamines are much less concentrated in the bridging sites but rather form a condensed layer around each polyelectrolyte. The diamines remain mostly isotropic, but have a slight tendency to align parallel to the rods in the condensed layer and perpendicular to the rods elsewhere. As the diamine length increases further $(\delta=20 \AA)$, however, a more complex structure appears, where the diamines are once again strongly concentrated in the bridging sites but also exhibit a high degree of alignment. Close to the rods the diamines orient themselves in parallel, and in the bridging sites they orient themselves perpendicular to the rods. We have explicitly verified that in the bridging sites the diamines are oriented such that both monovalent ends lie within the condensed layer of neighboring rods. Thus, longer diamines act as "linkers" between the rods, which accounts for the rise in electrostatic attraction and the resurgence of bundle stability.

Lastly, we consider the relationship between polyelectrolyte surface charge density $\sigma$ and bundle stability. Increasing $\sigma$ enhances the direct rod repulsion as well as the coupling between the rod and the condensing agent. Theoretical work [10, 11] predicts that the latter effect dominates: an effective attraction occurs when both the coupling parameter $\Xi=q^{2} \ell_{\mathrm{B}} / \mu$ and the Manning parameter $\xi=R / \mu$ are sufficiently large, where $q=2$ is the diamine valency, $\ell_{\mathrm{B}}=e^{2} /\left(4 \pi \varepsilon \varepsilon_{0} k_{\mathrm{B}} T\right)$ the Bjerrum length, and $\mu=\left(2 \pi q \ell_{\mathrm{B}} \sigma\right)^{-1}$ the Gouy-Chapman length. Both $\Xi$ and $\xi$ increase with $\sigma$, so that the net electrostatic attraction is enhanced at larger surface charge densities. There is another consideration, however: more highly charged rods require a higher concentration of diamines 


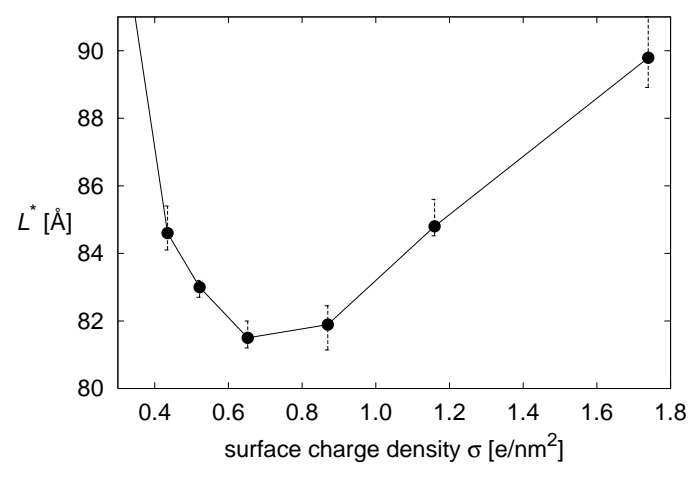

FIG. 4: Stable rod separation $L^{*}$ as a function of polyelectrolyte surface charge density $\sigma$, for $\delta=10 \AA$ at $60 \mathrm{mM}$ bulk diamine concentration. The curve demonstrates that increasing $\sigma$ can either increase or decrease bundle stability.

to maintain electroneutrality of the bundle, leading to an increase in the repulsive short-range contribution to the pressure. A priori, it is not clear which of these trendsenhanced electrostatic attractions, or larger excludedvolume repulsions - dominates, and there is experimental evidence supporting both scenarios. With diamines as the condensing agent, an increase in surface charge density from $0.303 \mathrm{e} / \mathrm{nm}^{2}$ to $0.343 \mathrm{e} / \mathrm{nm}^{2}$ dissolves the bundle, and a large increase in diamine concentration is needed to regain bundle stability [14]. But for certain divalent cations an increase in $\sigma$ from $0.343 \mathrm{e} / \mathrm{nm}^{2}$ to $0.457 \mathrm{e} / \mathrm{nm}^{2}$ [17] can enhance bundle stability [13]. Thus, the experimentally observed trend appears to depend on the condensing agent.

We shed some light on this situation by varying $\sigma$ in our simulations from $0.326 \mathrm{e} / \mathrm{nm}^{2}$ to $1.740 \mathrm{e} / \mathrm{nm}^{2}$, at constant diamine length $\delta=10 \AA$. For this $\delta$ the degree of alignment within the bundle is relatively low and partitioning of diamines between bundle and bulk is quite important, so variation of $L^{*}$ is a strong indication of bundle stability. At a fixed diamine concentration of $60 \mathrm{mM}, L^{*}$ indeed shows a strikingly nonmonotonic variation with $\sigma$ (Fig. 4). For small surface charge density the bundle stability increases with $\sigma$, as expected from the enhanced electrostatic coupling, yet for $\sigma \gtrsim 0.7 \mathrm{e} / \mathrm{nm}^{2}$ this trend is reversed, owing to the large number of neutralizing diamines within the bundle. This reversal suggests that by varying $\sigma$ over a sufficiently wide range, it may be possible to observe in a single set of experiments, with a single condensing agent, both of the trends which have previously been observed only separately.

It is worthwhile to comment on the five dimensionless parameters that characterize the system [18]. The coupling parameter $\Xi$ and the Manning parameter $\xi$ are convenient choices for two of these. Two further parameters account for the role of rod-rod separation and bulk diamine concentration. To account for diamine size,
Ref. 14] proposed $\psi \equiv \mu / \delta$, with the suggestion that $\psi>1$ is necessary for bundle formation. However, our demonstration that bundle stability is nonmonotonic in $\delta$ makes this choice of $\psi$ problematic. Moreover, the criterion $\mu / \delta>1$ predicts a dependence on temperature, valency, and dielectric constant that contradicts well-established trends for bundle stability. Instead, we suggest the parameter $\delta / a_{s}$, where $a_{s}$ is the charge separation on the polyelectrolyte surface. This choice was considered in Ref. [14], but disregarded because it did not vary significantly in the experiments. Here, we have varied $\delta / a_{s}$ over a much larger range. Interestingly, if this is done through variation of $\sigma$ (Fig. (4), the most stable condition indeed corresponds to $\delta / a_{s}=\mathcal{O}(1)$, i.e., the diamine charge separation matches the charge separation on the rod surface. However, we also note that this parameter cannot capture the nonmonotonicity observed as a function of $\delta$ [Fig. 2(b)], as it does not account for the alignment effects occurring for long diamine molecules.

In summary, we have clarified several mechanisms governing the role of condensing agents in polyelectrolyte bundling. We have also presented a unified picture for the dependence on surface charge density, combining seemingly conflicting experimental observations.

This material is based upon work supported by the National Science Foundation under CAREER Grant No. DMR-0346914 and Grant No. CTS-0120978 via the WaterCAMPWS Science and Technology Center.

* Corresponding author. E-mail: luijten@uiuc.edu

[1] J. X. Tang and P. A. Janmey, J. Biol. Chem. 271, 8556 (1996).

[2] A. P. Lyubartsev, J. X. Tang, P. A. Janmey, and L. Nordenskiöld, Phys. Rev. Lett. 81, 5465 (1998).

[3] R. W. Wilson and V. A. Bloomfield, Biochemistry 18, 2192 (1979).

[4] L. K. Sanders et al., Phys. Rev. Lett. 95, 108302 (2005).

[5] J. Ray and G. S. Manning, Langmuir 10, 2450 (1994).

[6] N. Grønbech-Jensen, R. J. Mashl, R. F. Bruinsma, and W. M. Gelbart, Phys. Rev. Lett. 78, 2477 (1997).

[7] B.-Y. Ha and A. J. Liu, Phys. Rev. Lett. 79, 1289 (1997).

[8] F. J. Solis and M. Olvera de la Cruz, Phys. Rev. E 60, 4496 (1999).

[9] B. I. Shklovskii, Phys. Rev. Lett. 82, 3268 (1999).

[10] A. Naji and R. R. Netz, Eur. Phys. J. E 13, 43 (2004).

[11] A. Naji, S. Jungblut, A. G. Moreira, and R. R. Netz, Physica A 352, 131 (2005).

[12] F. J. Solis and M. Olvera de la Cruz, Eur. Phys. J. E 4, 143 (2001).

[13] J. X. Tang, P. A. Janmey, A. Lyubartsev, and L. Nordenskiöld, Biophys. J. 83, 566 (2002).

[14] J. C. Butler, T. Angelini, J. X. Tang, and G. C. L. Wong, Phys. Rev. Lett. 91, 028301 (2003).

[15] In Ref. [13], variation of the surface charge was realized by exchanging M13 for the structurally identical fd virus.

[16] For each $\delta$ the threshold concentration is obtained 
through interpolation of simulation results for concentrations bracketing $C^{*}$, using histogram reweighting.

[17] These surface charge densities are calculated based upon the value reported in Ref. [14] and the difference between
M13 and fd.

[18] We assume that the diameter of the diamine beads can be absorbed in a redefinition of the rod radius [11]. 